\title{
Synergistic effects between biogenic ligands and a reductant in Fe
}

\section{acquisition from calcareous soil}

\author{
Walter D.C. Schenkeveld ${ }^{a^{*}}$, Zimeng Wang ${ }^{b}$, Daniel E. Giammar ${ }^{c}$ and Stephan M. Kraemer ${ }^{a^{*}}$ \\ ${ }^{a}$ Department of Environmental Geosciences Geosciences and Environmental Science Research \\ Network, University of Vienna, Vienna, Austria \\ ${ }^{\mathrm{b}}$ Department of Civil and Environmental Engineering, Stanford University, Stanford, California, United \\ States \\ ${ }^{c}$ Department of Energy, Environmental and Chemical Engineering, Washington University in St. Louis, \\ St. Louis, Missouri, United States \\ *Corresponding authors: walter.schenkeveld@univie.ac.at; stephan.kraemer@univie.ac.at
}

Figures: 2

Tables: 4

Pages: 12

\author{
Manuscript Submitted to \\ Environmental Science \& Technology \\ April 2016
}

\section{Supporting Information}

\section{Contents}

More detailed data description for soil interaction experiment with DFOB and ascorbate

Figure S1. Metal mobilization in the esculetin - ascorbate systems

Figure S2. Metal mobilization in the citrate - ascorbate systems

Table S1. Metal mobilization data presented in Figure 1 (DFOB)

Table S2. Metal mobilization data presented in Figure 2 (DMA)

Table S3. Metal mobilization data presented in Figure 3 and S1 (esculetin)

Table S4. Metal mobilization data presented in Figure 4 and S2 (citrate) 


\section{More detailed data description for soil interaction experiment with DFOB and ascorbate}

For $\mathrm{Mn}$ and Co mobilization from soil, a temporary synergistic effect was observed up to 24 and $48 \mathrm{~h}$, respectively. Mn mobilization by DFOB only was small (0.5 -1.4 $\mu \mathrm{M})$, for Co it was negligible $(>0.1 \mu \mathrm{M})$; $\mathrm{Mn}$ mobilization by ascorbate only was large, up to $37.1 \mu \mathrm{M}$ after $1 \mathrm{~h}$, Co mobilization by ascorbate only was small, up to $0.1 \mu \mathrm{M}$.

In the DFOB only treatment, Al was the most strongly mobilized metal, up to $24.5 \mu \mathrm{M}$; in the ascorbate only treatment Al mobilization was small $(<1.5 \mu \mathrm{M})$. The antagonistic effect in Al mobilization set in after $0.5 \mathrm{~h}$, reached a maximum of $5.2 \mu \mathrm{M}$ after $4 \mathrm{~h}$ and diminished slightly towards the end of the experiment. Despite the antagonistic effect, a factor two more Al than Fe was mobilized in the combined treatment.

Total metal mobilization increased from $10 \mu \mathrm{M}(0.25 \mathrm{~h})$ to $29 \mu \mathrm{M}(168 \mathrm{~h})$ in the DFOB only treatment, it increased from $29.7 \mu \mathrm{M}(0.25 \mathrm{~h})$ to $38 \mu \mathrm{M}(1 \mathrm{~h})$ and then decreased to $21 \mu \mathrm{M}(168 \mathrm{~h})$ in the ascorbate only treatment and increased from $51 \mu \mathrm{M}(0.25 \mathrm{~h})$ to a maximum of $60 \mu \mathrm{M}$ (4h) after which it decreased to $53 \mu \mathrm{M}(168 \mathrm{~h})$ in the combined treatment. Metal mobilization in the treatments with DFOB corresponded to a small fraction of the added ligand $(500 \mu \mathrm{M})$ : up to $6 \%$ in the DFOB only treatment and $12 \%$ in combined treatment. 

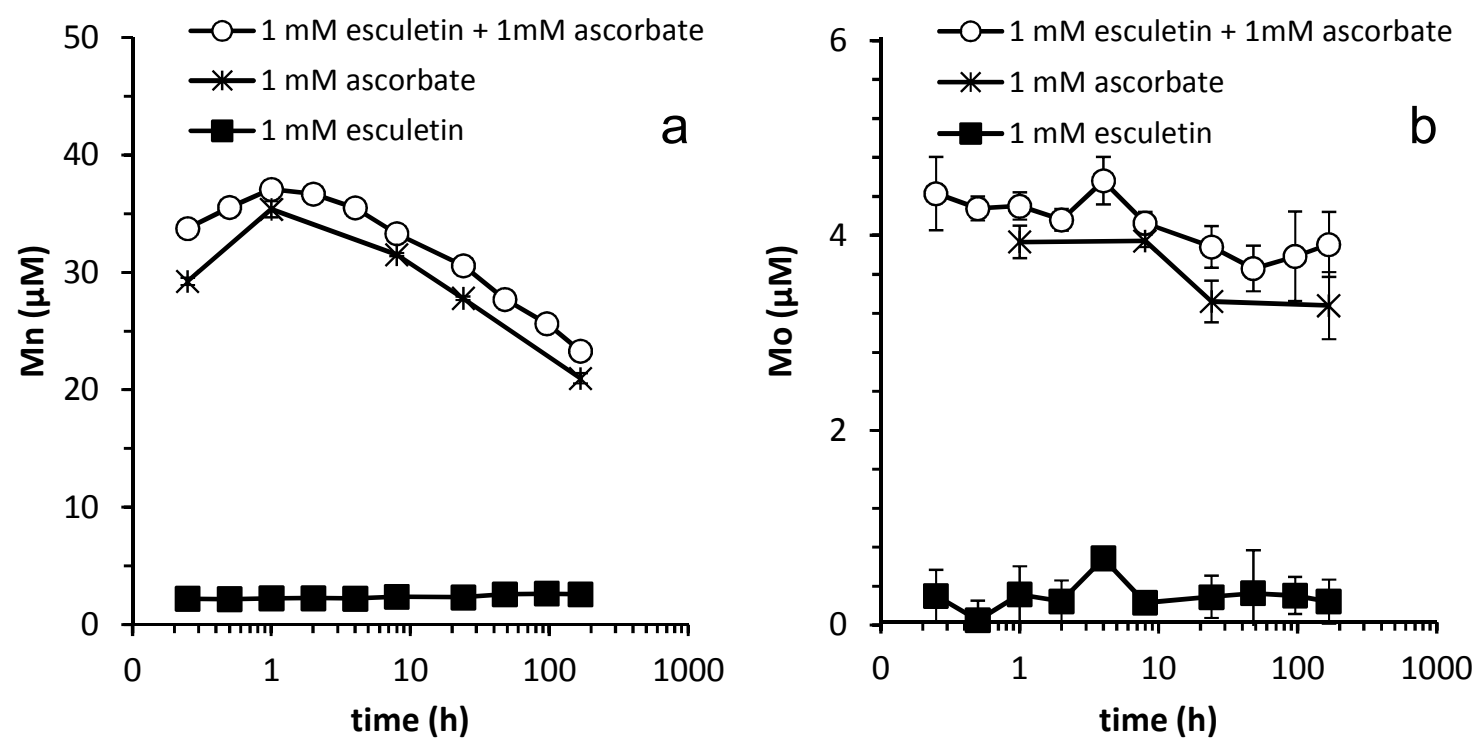

Figure S1: Mobilization of a) Mn, b) Mo from Santomera soil as a function of time by solutions containing $1 \mathrm{mM}$ esculetin and $1 \mathrm{mM}$ ascorbate, either separately or combined ( $\mathrm{SSR}=1 ; 10 \mathrm{mM} \mathrm{CaCl}$ ). The solutions contained a sterilant $\left(0.2 \mathrm{gl}^{-1}\right.$ bronopol) to prevent biodegradation. Error bars indicate standard deviations. 

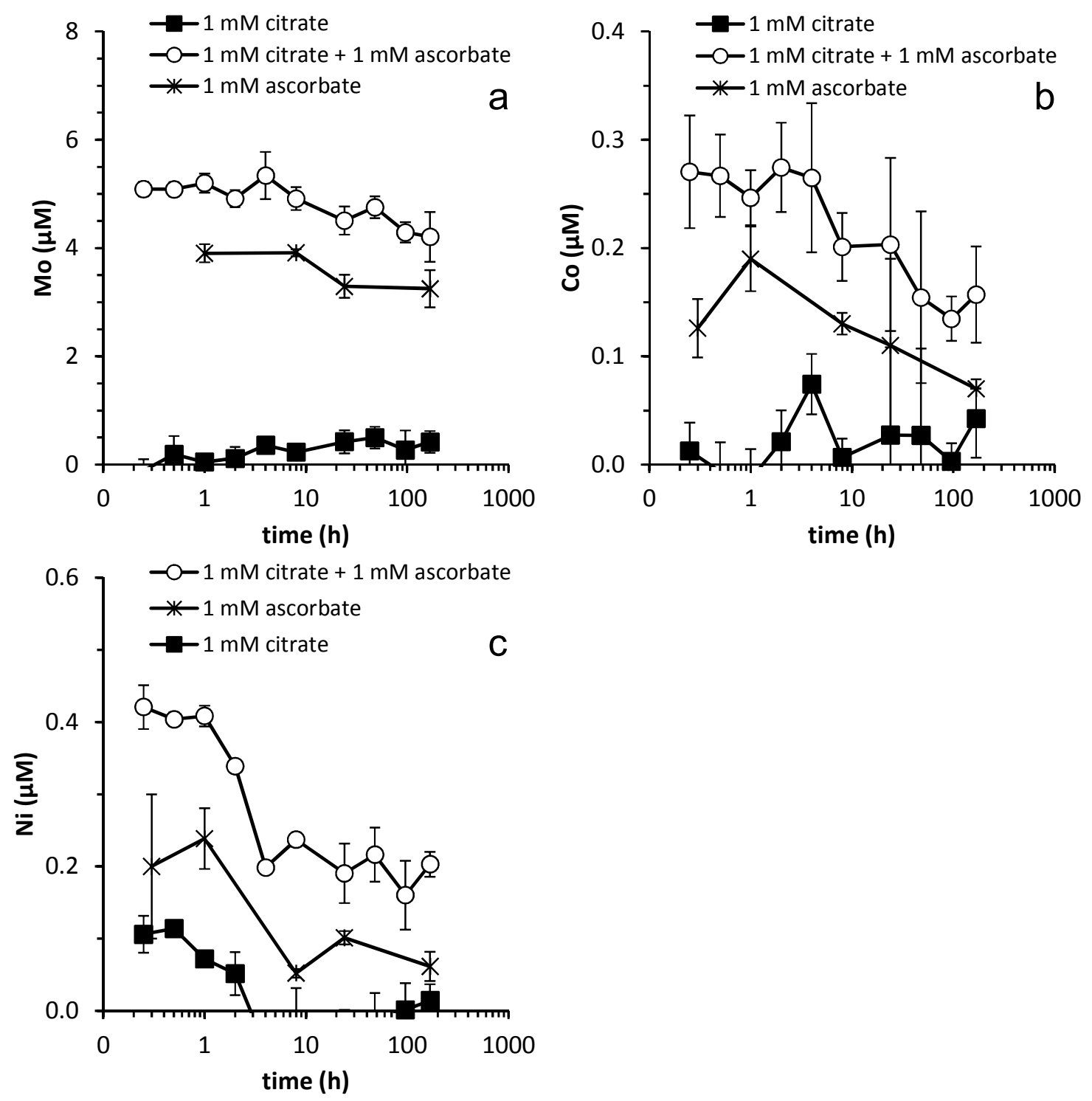

Figure S2: Mobilization of a) Mo, b) Co, and c) Ni from Santomera soil as a function of time by solutions containing $1 \mathrm{mM}$ citrate and $1 \mathrm{mM}$ ascorbate, either separately or combined ( $\mathrm{SSR}=1 ; 10 \mathrm{mM} \mathrm{CaCl}$ ). The solutions contained a sterilant $\left(0.2 \mathrm{~g} \mathrm{I}^{-1}\right.$ bronopol) to prevent biodegradation. Error bars indicate standard deviations. 
Table S1: Metal mobilization data presented in Figure 1 (DFOB)

\begin{tabular}{|c|c|c|c|c|c|c|}
\hline \multirow{2}{*}{$\begin{array}{c}\text { time } \\
\mathrm{h} \\
\end{array}$} & \multicolumn{2}{|c|}{ DFOB-only } & \multicolumn{2}{|c|}{ DFOB + ascorbate } & \multicolumn{2}{|c|}{ ascorbate } \\
\hline & $\begin{array}{c}\text { average } \\
\mu \mathrm{M}\end{array}$ & $\begin{array}{c}\text { stan.dev. } \\
\mu \mathrm{M}\end{array}$ & $\begin{array}{c}\text { average } \\
\mu \mathrm{M}\end{array}$ & $\begin{array}{c}\text { stan.dev. } \\
\mu \mathrm{M}\end{array}$ & $\begin{array}{c}\text { average } \\
\mu \mathrm{M}\end{array}$ & $\begin{array}{c}\text { stan.dev. } \\
\mu \mathrm{M}\end{array}$ \\
\hline 0.25 & 0.9 & 0.0 & 3.8 & 0.0 & 0.2 & 0.1 \\
\hline 0.5 & 0.7 & 0.0 & 3.9 & 0.0 & & \\
\hline 1 & 0.8 & 0.0 & 4.3 & 0.0 & 0.1 & 0.1 \\
\hline 2 & 1.1 & 0.1 & 5.0 & 0.1 & & \\
\hline 4 & 1.8 & 0.2 & 5.8 & 0.2 & & \\
\hline 8 & 2.2 & 0.1 & 6.6 & 0.1 & 0.0 & 0.2 \\
\hline 24 & 2.7 & 0.2 & 7.4 & 0.2 & 0.0 & 0.0 \\
\hline 48 & 3.3 & 0.2 & 7.8 & 0.2 & & \\
\hline 96 & 3.5 & 0.2 & 7.7 & 0.2 & & \\
\hline 168 & 5.2 & 0.3 & 9.2 & 0.3 & 0.0 & 0.1 \\
\hline
\end{tabular}

\begin{tabular}{ccccccc} 
Mn & \multicolumn{1}{c}{ DFOB + ascorbate } & \multicolumn{2}{c}{ ascorbate } \\
\hline time & \multicolumn{2}{c}{ DFOB-only } & \multicolumn{2}{c}{ DFan.dev. } & average & stan.dev. \\
& average & stan.dev. & average & stan. & stan \\
$\mathrm{h}$ & $\mu \mathrm{M}$ & $\mu \mathrm{M}$ & $\mu \mathrm{M}$ & $\mu \mathrm{M}$ & $\mu \mathrm{M}$ & $\mu \mathrm{M}$ \\
\hline 0.25 & 1.2 & 0.0 & 38.1 & 0.0 & 29.2 & 0.3 \\
0.5 & 1.4 & 0.1 & 40.5 & 0.1 & & \\
1 & 1.4 & 0.0 & 41.5 & 0.0 & 37.1 & 0.1 \\
2 & 1.5 & 0.0 & 41.8 & 0.0 & & \\
4 & 1.0 & 0.2 & 38.3 & 0.2 & & \\
8 & 0.7 & 0.1 & 34.7 & 0.1 & 32.8 & 0.1 \\
24 & 0.5 & 0.0 & 29.6 & 0.0 & 29.2 & 0.5 \\
48 & 1.0 & 0.0 & 25.6 & 0.0 & & \\
96 & 0.2 & 0.1 & 22.5 & 0.1 & & \\
168 & 1.2 & 0.1 & 24.2 & 0.1 & 20.7 & 0.1 \\
\hline
\end{tabular}

Co

\begin{tabular}{ccccccc}
\hline time & \multicolumn{2}{c}{ DFOB-only } & \multicolumn{2}{c}{ DFOB + ascorbate } & \multicolumn{2}{c}{ ascorbate } \\
& average & stan.dev. & average & stan.dev. & average & stan.dev. \\
$\mathrm{h}$ & $\mu \mathrm{M}$ & $\mu \mathrm{M}$ & $\mu \mathrm{M}$ & $\mu \mathrm{M}$ & $\mu \mathrm{M}$ & $\mu \mathrm{M}$ \\
\hline 0.25 & -0.01 & 0.04 & 0.53 & 0.04 & 0.13 & 0.03 \\
0.5 & -0.02 & 0.04 & 0.52 & 0.04 & & \\
1 & 0.03 & 0.04 & 0.54 & 0.04 & 0.14 & 0.04 \\
2 & 0.04 & 0.04 & 0.51 & 0.04 & & \\
4 & 0.00 & 0.03 & 0.48 & 0.03 & & \\
8 & 0.06 & 0.05 & 0.35 & 0.05 & 0.15 & 0.01 \\
24 & 0.01 & 0.03 & 0.28 & 0.03 & 0.14 & 0.03 \\
48 & 0.00 & 0.04 & 0.16 & 0.04 & & \\
96 & 0.00 & 0.05 & 0.12 & 0.05 & & \\
168 & 0.04 & 0.06 & 0.13 & 0.06 & 0.09 & 0.05 \\
\hline
\end{tabular}




\begin{tabular}{ccccccc} 
Al & \multicolumn{1}{c}{ DFOB + ascorbate } & \multicolumn{2}{c}{ ascorbate } \\
\hline time & \multicolumn{2}{c}{ DFOB-only } & \multicolumn{2}{c}{ DFan. } \\
& average & stan.dev. & average & stan.dev. & average & stan.dev. \\
$\mathrm{h}$ & $\mu \mathrm{M}$ & $\mu \mathrm{M}$ & $\mu \mathrm{M}$ & $\mu \mathrm{M}$ & $\mu \mathrm{M}$ & $\mu \mathrm{M}$ \\
\hline 0.25 & 8.5 & 0.4 & 8.6 & 0.4 & & \\
0.5 & 11.4 & 0.3 & 9.0 & 0.3 & & \\
1 & 13.5 & 0.3 & 10.6 & 0.3 & 0.5 & 1.1 \\
2 & 15.8 & 0.3 & 13.0 & 0.3 & & \\
4 & 20.9 & 0.9 & 15.7 & 0.9 & & \\
8 & 23.3 & 1.5 & 18.5 & 1.5 & 1.4 & 0.4 \\
24 & 24.2 & 1.0 & 19.8 & 1.0 & 1.0 & 0.9 \\
48 & 24.5 & 1.0 & 19.3 & 1.0 & & \\
96 & 22.3 & 0.9 & 18.6 & 0.9 & & \\
168 & 22.8 & 1.2 & 19.6 & 1.2 & 0.0 & 1.2 \\
\hline
\end{tabular}


Table S2: Metal mobilization data presented in Figure 2 (DMA)

\begin{tabular}{ccccccc} 
Fe & \multicolumn{10}{c}{ DMA-only } & \multicolumn{2}{c}{ DMA + ascorbate } & \multicolumn{2}{c}{ ascorbate } \\
\hline time & \multicolumn{2}{c}{ DMAn.dev. } & average & stan.dev. & average & stan.dev. \\
& average & stan.den & $\mu \mathrm{M}$ & $\mu \mathrm{M}$ \\
$\mathrm{h}$ & $\mu \mathrm{M}$ & $\mu \mathrm{M}$ & $\mu \mathrm{M}$ & $\mu \mathrm{M}$ & $\mu \mathrm{M}$ & 0.1 \\
\hline 0.25 & 3.1 & 0.4 & 9.9 & 0.4 & 0.2 & 0.1 \\
0.5 & 4.2 & 0.1 & 13.3 & 0.2 & & \\
1 & 5.9 & 0.1 & 15.6 & 0.2 & 0.1 & 0.1 \\
2 & 8.5 & 0.2 & 15.5 & 0.1 & & \\
4 & 12.4 & 0.2 & 13.1 & 0.4 & & \\
8 & 16.3 & 0.3 & 10.2 & 0.1 & 0.0 & 0.2 \\
24 & 20.0 & 0.2 & 6.0 & 0.2 & 0.0 & 0.0 \\
48 & 18.6 & 0.2 & 3.9 & 0.0 & & \\
96 & 14.8 & 0.2 & 2.2 & 0.1 & & \\
168 & 10.9 & 0.2 & 1.8 & 0.5 & 0.0 & 0.1 \\
\hline
\end{tabular}

\begin{tabular}{|c|c|c|c|c|c|c|}
\hline \multirow{2}{*}{$\begin{array}{c}\text { time } \\
\mathrm{h}\end{array}$} & \multicolumn{2}{|c|}{ DMA-only } & \multicolumn{2}{|c|}{ DMA + ascorbate } & \multicolumn{2}{|c|}{ ascorbate } \\
\hline & $\begin{array}{c}\text { average } \\
\mu \mathrm{M}\end{array}$ & $\begin{array}{c}\text { stan.dev. } \\
\mu \mathrm{M}\end{array}$ & $\begin{array}{c}\text { average } \\
\mu \mathrm{M}\end{array}$ & $\begin{array}{c}\text { stan.dev. } \\
\mu \mathrm{M}\end{array}$ & $\begin{array}{c}\text { average } \\
\mu \mathrm{M}\end{array}$ & $\begin{array}{c}\text { stan.dev. } \\
\mu \mathrm{M}\end{array}$ \\
\hline 0.25 & 2.2 & 0.1 & 2.6 & 0.0 & 0.0 & 0.1 \\
\hline 0.5 & 2.9 & 0.1 & 3.2 & 0.1 & & \\
\hline 1 & 3.6 & 0.0 & 4.0 & 0.1 & 0.1 & 0.1 \\
\hline 2 & 4.7 & 0.1 & 4.9 & 0.0 & & \\
\hline 4 & 6.1 & 0.0 & 6.2 & 0.1 & & \\
\hline 8 & 7.6 & 0.0 & 7.7 & 0.0 & 0.0 & 0.1 \\
\hline 24 & 10.9 & 0.1 & 10.6 & 0.1 & 0.1 & 0.0 \\
\hline 48 & 13.4 & 0.2 & 12.9 & 0.1 & & \\
\hline 96 & 16.5 & 0.2 & 15.1 & 0.0 & & \\
\hline 168 & 18.7 & 0.2 & 16.6 & 0.4 & 0.0 & 0.0 \\
\hline
\end{tabular}

\begin{tabular}{ccccccc}
$\mathbf{N i}$ & \multicolumn{1}{c}{ DMA-only } & \multicolumn{2}{c}{ DMA + ascorbate } & \multicolumn{2}{c}{ ascorbate } \\
\hline time & \multicolumn{2}{c}{ DMAan.dev. } & average & stan.dev. \\
& average & stan.dev. & average & stan.dev & s. \\
$\mathrm{h}$ & $\mu \mathrm{M}$ & $\mu \mathrm{M}$ & $\mu \mathrm{M}$ & $\mu \mathrm{M}$ & $\mu \mathrm{M}$ & $\mu \mathrm{M}$ \\
\hline 0.25 & 0.6 & 0.0 & 4.4 & 0.0 & 0.2 & 0.1 \\
0.5 & 0.8 & 0.1 & 5.4 & 0.0 & & \\
1 & 1.0 & 0.1 & 6.6 & 0.1 & 0.2 & 0.0 \\
2 & 1.5 & 0.0 & 7.7 & 0.1 & & \\
4 & 1.9 & 0.0 & 8.9 & 0.1 & & \\
8 & 2.4 & 0.1 & 9.9 & 0.0 & 0.1 & 0.0 \\
24 & 3.5 & 0.1 & 11.7 & 0.1 & 0.1 & 0.0 \\
48 & 4.4 & 0.1 & 12.9 & 0.1 & & \\
96 & 5.5 & 0.1 & 13.7 & 0.1 & & \\
168 & 6.9 & 0.1 & 14.7 & 0.1 & 0.2 & 0.0 \\
\hline
\end{tabular}


Co

\begin{tabular}{ccccccc}
\hline time & \multicolumn{2}{c}{ DMA-only } & \multicolumn{2}{c}{ DMA + ascorbate } & \multicolumn{2}{c}{ ascorbate } \\
& average & stan.dev. & average & stan.dev. & average & stan.dev. \\
$\mathrm{h}$ & $\mu \mathrm{M}$ & $\mu \mathrm{M}$ & $\mu \mathrm{M}$ & $\mu \mathrm{M}$ & $\mu \mathrm{M}$ & $\mu \mathrm{M}$ \\
\hline 0.25 & 0.2 & 0.0 & 9.8 & 0.1 & 0.1 & 0.0 \\
0.5 & 0.2 & 0.0 & 10.6 & 0.2 & & \\
1 & 0.3 & 0.0 & 11.4 & 0.1 & 0.2 & 0.0 \\
2 & 0.4 & 0.0 & 12.0 & 0.1 & & \\
4 & 0.5 & 0.1 & 12.6 & 0.1 & & \\
8 & 0.6 & 0.0 & 12.8 & 0.0 & 0.1 & 0.0 \\
24 & 1.4 & 0.0 & 13.6 & 0.1 & 0.1 & 0.0 \\
48 & 2.3 & 0.0 & 14.8 & 0.1 & & \\
96 & 4.2 & 0.1 & 16.4 & 0.1 & & 0.0 \\
168 & 6.8 & 0.0 & 18.3 & 0.9 & 0.1 & 0.0 \\
\hline
\end{tabular}

Mn

\begin{tabular}{ccccccc}
\hline time & \multicolumn{2}{c}{ DMA-only } & \multicolumn{2}{c}{ DMA + ascorbate } & \multicolumn{2}{c}{ ascorbate } \\
& average & stan.dev. & average & stan.dev. & average & stan.dev. \\
$\mathrm{h}$ & $\mu \mathrm{M}$ & $\mu \mathrm{M}$ & $\mu \mathrm{M}$ & $\mu \mathrm{M}$ & $\mu \mathrm{M}$ & $\mu \mathrm{M}$ \\
\hline 0.25 & 1.2 & 0.1 & 39.9 & 0.2 & 29.2 & 0.3 \\
0.5 & 1.2 & 0.0 & 38.5 & 0.1 & & \\
1 & 1.1 & 0.0 & 36.9 & 0.6 & 31.9 & 0.3 \\
2 & 1.1 & 0.1 & 34.7 & 0.3 & & \\
4 & 0.9 & 0.0 & 32.2 & 0.3 & & \\
8 & 0.7 & 0.0 & 28.7 & 0.2 & 26.5 & 0.3 \\
24 & 0.6 & 0.1 & 24.4 & 0.4 & 23.3 & 0.4 \\
48 & 0.8 & 0.1 & 22.2 & 0.1 & & \\
96 & -0.2 & 0.1 & 18.5 & 0.1 & & \\
168 & 0.7 & 0.1 & 19.6 & 2.0 & 17.5 & 0.6 \\
\hline
\end{tabular}

Zn

\begin{tabular}{ccccccc}
\hline time & \multicolumn{2}{c}{ DMA-only } & \multicolumn{2}{c}{ DMA + ascorbate } & \multicolumn{2}{c}{ ascorbate } \\
& average & stan.dev. & average & stan.dev. & average & stan.dev. \\
$\mathrm{h}$ & $\mu \mathrm{M}$ & $\mu \mathrm{M}$ & $\mu \mathrm{M}$ & $\mu \mathrm{M}$ & $\mu \mathrm{M}$ & $\mu \mathrm{M}$ \\
\hline 0.25 & 1.4 & 0.2 & 1.8 & 0.1 & 0.1 & 0.0 \\
0.5 & 1.5 & 0.0 & 2.1 & 0.1 & & \\
1 & 1.6 & 0.0 & 2.1 & 0.0 & 0.0 & 0.0 \\
2 & 1.9 & 0.1 & 2.3 & 0.0 & & \\
4 & 2.2 & 0.1 & 2.6 & 0.1 & & \\
8 & 2.5 & 0.0 & 2.5 & 0.1 & 0.0 & 0.0 \\
24 & 2.7 & 0.0 & 2.3 & 0.0 & 0.0 & 0.0 \\
48 & 2.7 & 0.0 & 1.8 & 0.1 & & \\
96 & 2.4 & 0.1 & 1.3 & 0.0 & & 0.0 \\
168 & 2.3 & 0.0 & 0.7 & 0.1 & 0.0 & \\
\hline
\end{tabular}


Table S3: Metal mobilization data presented in Figure 3 and S1 (esculetin)

\begin{tabular}{|c|c|c|c|c|c|c|}
\hline \multirow{2}{*}{$\begin{array}{c}\text { time } \\
\mathrm{h}\end{array}$} & \multicolumn{2}{|c|}{ esculetin-only } & \multicolumn{2}{|c|}{ esculetine + ascorbate } & \multicolumn{2}{|c|}{ ascorbate } \\
\hline & $\begin{array}{c}\text { average } \\
\mu \mathrm{M}\end{array}$ & $\begin{array}{c}\text { stan.dev. } \\
\mu \mathrm{M}\end{array}$ & $\begin{array}{c}\text { average } \\
\mu \mathrm{M}\end{array}$ & $\begin{array}{c}\text { stan.dev. } \\
\mu \mathrm{M}\end{array}$ & $\begin{array}{c}\text { average } \\
\mu \mathrm{M}\end{array}$ & $\begin{array}{c}\text { stan.dev. } \\
\mu \mathrm{M}\end{array}$ \\
\hline 0.25 & 0.10 & 0.02 & 0.53 & 0.02 & 0.19 & 0.14 \\
\hline 0.5 & 0.11 & 0.02 & 0.53 & 0.02 & & \\
\hline 1 & 0.12 & 0.02 & 0.48 & 0.03 & 0.06 & 0.02 \\
\hline 2 & 0.10 & 0.02 & 0.34 & 0.02 & & \\
\hline 4 & 0.13 & 0.05 & 0.31 & 0.04 & & \\
\hline 8 & 0.08 & 0.04 & 0.22 & 0.04 & 0.02 & 0.03 \\
\hline 24 & 0.07 & 0.04 & 0.12 & 0.04 & 0.01 & 0.03 \\
\hline 48 & 0.12 & 0.03 & 0.12 & 0.05 & & \\
\hline 96 & 0.02 & 0.01 & 0.06 & 0.01 & & \\
\hline 168 & 0.01 & 0.02 & 0.06 & 0.02 & -0.02 & 0.02 \\
\hline
\end{tabular}

\begin{tabular}{|c|c|c|c|c|c|c|}
\hline \multirow{2}{*}{$\begin{array}{c}\text { time } \\
\mathrm{h}\end{array}$} & \multicolumn{2}{|c|}{ esculetin-only } & \multicolumn{2}{|c|}{ esculetine + ascorbate } & \multicolumn{2}{|c|}{ ascorbate } \\
\hline & $\begin{array}{c}\text { average } \\
\mu \mathrm{M}\end{array}$ & $\begin{array}{c}\text { stan.dev. } \\
\mu \mathrm{M}\end{array}$ & $\begin{array}{c}\text { average } \\
\mu \mathrm{M}\end{array}$ & $\begin{array}{c}\text { stan.dev. } \\
\mu \mathrm{M}\end{array}$ & $\begin{array}{c}\text { average } \\
\mu \mathrm{M}\end{array}$ & $\begin{array}{c}\text { stan.dev } \\
\mu \mathrm{M}\end{array}$ \\
\hline 0.25 & 2.2 & 0.1 & 33.8 & 0.5 & 29.2 & 0.3 \\
\hline 0.5 & 2.2 & 0.0 & 35.5 & 0.3 & & \\
\hline 1 & 2.2 & 0.0 & 37.1 & 0.0 & 35.4 & 0.7 \\
\hline 2 & 2.3 & 0.0 & 36.7 & 0.2 & & \\
\hline 4 & 2.2 & 0.0 & 35.5 & 0.3 & & \\
\hline 8 & 2.4 & 0.0 & 33.3 & 0.3 & 31.5 & 0.1 \\
\hline 24 & 2.3 & 0.2 & 30.6 & 0.1 & 27.8 & 0.1 \\
\hline 48 & 2.6 & 0.1 & 27.7 & 0.3 & & \\
\hline 96 & 2.6 & 0.1 & 25.6 & 0.7 & & \\
\hline 168 & 2.6 & 0.1 & 23.3 & 0.2 & 21.0 & 0.4 \\
\hline
\end{tabular}

\begin{tabular}{ccccccc} 
Mo & \multicolumn{1}{c}{ esculetin-only } & \multicolumn{2}{c}{ esculetine + ascorbate } & \multicolumn{2}{c}{ ascorbate } \\
time & \multicolumn{2}{c}{ escan.dev. } & average & stan.dev. \\
& average & stan.dev. & average & stan.den & $\mu \mathrm{M}$ & $\mu \mathrm{M}$ \\
$\mathrm{h}$ & $\mu \mathrm{M}$ & $\mu \mathrm{M}$ & $\mu \mathrm{M}$ & $\mu \mathrm{M}$ & & \\
\hline 0.25 & 0.3 & 0.3 & 4.4 & 0.4 & & \\
0.5 & 0.0 & 0.2 & 4.2 & 0.1 & & 0.2 \\
1 & 0.3 & 0.3 & 4.3 & 0.1 & 3.9 & \\
2 & 0.2 & 0.2 & 4.1 & 0.1 & & \\
4 & 0.7 & 0.0 & 4.5 & 0.2 & & \\
8 & 0.2 & 0.1 & 4.1 & 0.1 & 3.9 & 0.1 \\
24 & 0.3 & 0.2 & 3.9 & 0.2 & 3.3 & 0.2 \\
48 & 0.3 & 0.4 & 3.6 & 0.2 & & \\
96 & 0.3 & 0.2 & 3.8 & 0.5 & & \\
168 & 0.2 & 0.2 & 3.9 & 0.3 & 3.2 & 0.3 \\
\hline
\end{tabular}


Table S4: Metal mobilization data presented in Figure 4 and S2 (citrate)

\begin{tabular}{|c|c|c|c|c|c|c|}
\hline \multirow{2}{*}{$\begin{array}{c}\text { time } \\
\mathrm{h}\end{array}$} & \multicolumn{2}{|c|}{ citrate } & \multicolumn{2}{|c|}{ citrate + ascorbate } & \multicolumn{2}{|c|}{ ascorbate } \\
\hline & $\begin{array}{c}\text { average } \\
\mu \mathrm{M}\end{array}$ & $\begin{array}{c}\text { stan.dev. } \\
\mu \mathrm{M}\end{array}$ & $\begin{array}{c}\text { average } \\
\mu \mathrm{M}\end{array}$ & $\begin{array}{c}\text { stan.dev. } \\
\mu \mathrm{M}\end{array}$ & $\begin{array}{c}\text { average } \\
\mu \mathrm{M}\end{array}$ & $\begin{array}{c}\text { stan.dev. } \\
\mu \mathrm{M}\end{array}$ \\
\hline 0.25 & 0.10 & 0.02 & 0.53 & 0.02 & 0.19 & 0.14 \\
\hline 0.5 & 0.11 & 0.02 & 0.53 & 0.02 & & \\
\hline 1 & 0.12 & 0.02 & 0.48 & 0.03 & 0.06 & 0.02 \\
\hline 2 & 0.10 & 0.02 & 0.34 & 0.02 & & \\
\hline 4 & 0.13 & 0.05 & 0.31 & 0.04 & & \\
\hline 8 & 0.08 & 0.04 & 0.22 & 0.04 & 0.02 & 0.03 \\
\hline 24 & 0.07 & 0.04 & 0.12 & 0.04 & 0.01 & 0.03 \\
\hline 48 & 0.12 & 0.03 & 0.12 & 0.05 & & \\
\hline 96 & 0.02 & 0.01 & 0.06 & 0.01 & & \\
\hline 168 & 0.01 & 0.02 & 0.06 & 0.02 & -0.02 & 0.02 \\
\hline
\end{tabular}

\begin{tabular}{ccccccc} 
Al & \multicolumn{10}{c}{} \\
\hline time & \multicolumn{2}{c}{ citrate } & \multicolumn{2}{c}{ citrate + ascorbate } & \multicolumn{2}{c}{ ascorbate } \\
& average & stan.dev. & average & stan.dev. & average & stan.dev. \\
$\mathrm{h}$ & $\mu \mathrm{M}$ & $\mu \mathrm{M}$ & $\mu \mathrm{M}$ & $\mu \mathrm{M}$ & $\mu \mathrm{M}$ & $\mu \mathrm{M}$ \\
\hline 0.25 & 6.9 & 0.3 & 3.8 & 0.3 & & \\
0.5 & 7.0 & 0.5 & 4.2 & 0.1 & & \\
1 & 5.8 & 0.1 & 3.9 & 0.1 & 0.0 & 0.1 \\
2 & 5.3 & 0.1 & 3.5 & 0.1 & & \\
4 & 4.2 & 0.3 & 3.3 & 0.3 & & \\
8 & 3.7 & 0.4 & 3.2 & 0.4 & -0.2 & 0.1 \\
24 & 3.0 & 0.0 & 2.4 & 0.0 & 0.3 & 0.2 \\
48 & 1.8 & 0.1 & 2.4 & 1.2 & & \\
96 & 1.6 & 0.4 & 2.0 & 0.1 & & \\
168 & 1.4 & 0.2 & 1.9 & 0.1 & -0.1 & 0.2 \\
\hline
\end{tabular}

\begin{tabular}{ccccccc} 
Mo & \multicolumn{1}{c}{ citrate + ascorbate } & \multicolumn{2}{c}{ ascorbate } \\
\hline time & \multicolumn{2}{c}{ citrate } & \multicolumn{2}{c}{ citrase } \\
& average & stan.dev. & average & stan.dev. & average & stan.dev. \\
$\mathrm{h}$ & $\mu \mathrm{M}$ & $\mu \mathrm{M}$ & $\mu \mathrm{M}$ & $\mu \mathrm{M}$ & $\mu \mathrm{M}$ & $\mu \mathrm{M}$ \\
\hline 0.25 & -0.1 & 0.2 & 5.1 & 0.1 & & \\
0.5 & 0.2 & 0.3 & 5.1 & 0.1 & & \\
1 & 0.0 & 0.1 & 5.2 & 0.2 & 3.9 & 0.2 \\
2 & 0.1 & 0.2 & 4.9 & 0.2 & & \\
4 & 0.4 & 0.0 & 5.3 & 0.4 & & \\
8 & 0.2 & 0.0 & 4.9 & 0.2 & 3.9 & 0.1 \\
24 & 0.4 & 0.2 & 4.5 & 0.3 & 3.3 & 0.2 \\
48 & 0.5 & 0.2 & 4.8 & 0.2 & & \\
96 & 0.3 & 0.4 & 4.3 & 0.2 & & \\
168 & 0.4 & 0.2 & 4.2 & 0.5 & 3.2 & 0.3 \\
\hline
\end{tabular}


Co

\begin{tabular}{ccccccc}
\hline time & \multicolumn{2}{c}{ citrate } & \multicolumn{2}{c}{ citrate + ascorbate } & \multicolumn{2}{c}{ ascorbate } \\
& average & stan.dev. & average & stan.dev. & average & stan.dev. \\
$\mathrm{h}$ & $\mu \mathrm{M}$ & $\mu \mathrm{M}$ & $\mu \mathrm{M}$ & $\mu \mathrm{M}$ & $\mu \mathrm{M}$ & $\mu \mathrm{M}$ \\
\hline 0.25 & 0.01 & 0.03 & 0.27 & 0.05 & 0.1 & 0.0 \\
0.5 & -0.01 & 0.03 & 0.27 & 0.04 & & \\
1 & -0.01 & 0.03 & 0.25 & 0.03 & 0.19 & 0.03 \\
2 & 0.02 & 0.03 & 0.27 & 0.04 & & \\
4 & 0.07 & 0.03 & 0.26 & 0.07 & & \\
8 & 0.01 & 0.02 & 0.20 & 0.03 & 0.13 & 0.01 \\
24 & 0.03 & 0.08 & 0.20 & 0.08 & 0.11 & 0.08 \\
48 & 0.03 & 0.08 & 0.15 & 0.08 & & \\
96 & 0.00 & 0.02 & 0.13 & 0.02 & & \\
168 & 0.04 & 0.04 & 0.16 & 0.04 & 0.07 & 0.00 \\
\hline
\end{tabular}

Ni

\begin{tabular}{ccccccc}
\hline time & \multicolumn{2}{c}{ citrate } & \multicolumn{2}{c}{ citrate + ascorbate } & \multicolumn{2}{c}{ ascorbate } \\
& average & stan.dev. & average & stan.dev. & average & stan.dev. \\
$\mathrm{h}$ & $\mu \mathrm{M}$ & $\mu \mathrm{M}$ & $\mu \mathrm{M}$ & $\mu \mathrm{M}$ & $\mu \mathrm{M}$ & $\mu \mathrm{M}$ \\
\hline 0.25 & 0.11 & 0.03 & 0.42 & 0.03 & 0.2 & 0.1 \\
0.5 & 0.11 & 0.01 & 0.40 & 0.01 & & \\
1 & 0.07 & 0.01 & 0.41 & 0.01 & 0.24 & 0.04 \\
2 & 0.05 & 0.03 & 0.34 & 0.01 & & \\
4 & -0.05 & 0.01 & 0.20 & 0.01 & & \\
8 & -0.01 & 0.04 & 0.24 & 0.01 & 0.05 & 0.01 \\
24 & -0.03 & 0.04 & 0.19 & 0.04 & 0.10 & 0.01 \\
48 & -0.02 & 0.04 & 0.22 & 0.04 & & \\
96 & 0.00 & 0.04 & 0.16 & 0.05 & & \\
168 & 0.01 & 0.02 & 0.20 & 0.02 & 0.06 & 0.02 \\
\hline
\end{tabular}


1. Siebner-Freibach, H.; Hadar, Y.; Yariv, S.; Lapides, I.; Chen, Y., Thermos pectroscopic study of the adsorption mechanism of the hydroxamic siderophore ferrioxamine B by calcium montmorillonite. J. Agric. Food Chem. 2006, 54, (4), 1399-1408.

2. Siebner-Freibach, H.; Hadar, Y.; Chen, Y., Interaction of iron chelating agents with clay minerals. Soil Sci. Soc. Am. J. 2004, 68, (2), 470-480. 